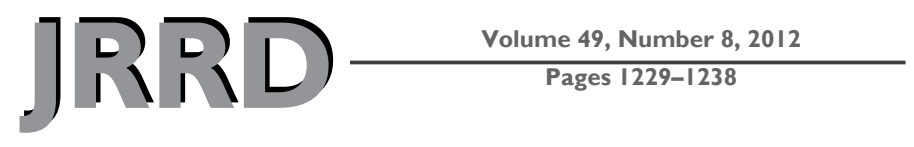

\title{
Device to monitor sock use in people using prosthetic limbs: Technical report
}

\author{
Joan E. Sanders, PhD; ${ }^{*}$ Revathi Murthy, MSE; ${ }^{1}$ John C. Cagle, BSE; ${ }^{1}$ Katheryn J. Allyn, CPO; ${ }^{1}$ Reid H. Phil- \\ lips, BSE; ${ }^{1}$ Brian P. Otis, PhD $^{2}$ \\ Departments of ${ }^{1}$ Bioengineering and ${ }^{2}$ Electrical Engineering, University of Washington, Seattle, WA
}

\begin{abstract}
A device using radio frequency identification (RFID) technology was developed to continuously monitor sock use in people who use prosthetic limbs. RFID tags were placed on prosthetic socks worn by subjects with transtibial limb loss, and a high-frequency RFID reader and antenna were placed in a portable unit mounted to the outside of the prosthetic socket. Bench testing showed the device to have a maximum read range between $5.6 \mathrm{~cm}$ and $12.7 \mathrm{~cm}$, depending on the RFID tag used. Testing in a laboratory setting on three participants with transtibial amputation showed that the device correctly monitored sock presence during sitting, standing, and walking activity when one or two socks were worn but was less reliable when more socks were used. Accurate detection was sensitive to orientation of the tag relative to the reader, presence of carbon fiber in the prosthetic socket, pistoning of the limb in the socket, and overlap among the tags. Use of ultra-highfrequency RFID may overcome these limitations. With improvements, the technology may prove useful to practitioners prescribing volume accommodation strategies for patients by providing information about sock use between clinical visits, including timing and consistency of daily sock-ply changes.
\end{abstract}

Key words: accommodation, diurnal, interface mechanics, limb-socket, prosthesis, radio frequency, rehabilitation, residual limb, sock monitor, transtibial amputee, volume.

\section{BACKGROUND AND AIM}

People with limb loss are commonly advised to add or remove socks to accommodate changes in the size of their residual limb over the day [1]. Part of the challenge for a practitioner helping a patient solve limb volume management problems is that information about the patient's sock use outside of the clinic is limited to verbal input from the patient. Particularly for people with cognitive issues, an accurate account of how many and which socks were worn each morning and afternoon over a period of days or weeks is difficult to create. The practitioner is typically provided with limited information from which to optimize prescription. Though it has yet to be studied through scientific research investigation, this limitation may in some cases extend the fitting or socket modification process, putting the residual limb at risk of injury.

The purpose of this technical note is to describe a device for continuous monitoring of the number and type (i.e., model, ply) of socks worn by a prosthesis user. The long-term goal is a small unobtrusive instrument that a clinician mounts on the patient's prosthesis to record sock use continuously between clinical visits. Potentially,

\footnotetext{
Abbreviations: FSR = force sensing resistor, HF = highfrequency, ID = identification, $\mathrm{PTB}$ = patellar-tendon bearing, RFID = radio frequency identification, $\mathrm{SD}=$ secure digital, $\mathrm{UHF}=$ ultra-high-frequency.

*Address all correspondence to Joan E. Sanders, PhD; Department of Bioengineering, Box 355061, Foege N430J, 3720 15th Ave, NE, University of Washington, Seattle, WA 98195-5061; 206-221-5872; fax: 206-616-2509.

Email: jsanders@u.washington.edu http://dx.doi.org/10.1682/JRRD.2011.09.0169
} 
the instrument could be extended to instruct a patient when socks need to be added. After a recording period, the practitioner downloads collected data to view a record of daily sock use, when sock changes were made, and how consistently the patient performed accommodation. This information may help the practitioner enhance prescription [2]; it is new information not currently available. A practitioner may gain insight into differences between a patient's weekday and weekend volume changes, for example. In this technical note, we describe the design of a prototype instrument to monitor sock presence, results from subject testing, and recommendations for further development.

\section{METHODS AND RESULTS}

\section{Device Design}

The sock monitor uses radio frequency identification (RFID) technology to detect sock presence. Passive RFID tags ( $<0.1 \mathrm{~mm}$ thick) are taped to the user's prosthetic socks (one tag per sock), and a tag reader is mounted on the external socket surface (Figure 1). The reader, via its antenna, communicates wirelessly to the tags to determine which tags are present. Because it is desirable to detect only socks inside the socket, high-frequency (HF) radio communication (13.56 MHz) is used [3].

HF RFID has a relatively short detection range (less than $\sim 14 \mathrm{~cm}$ ), consistent with the needs of the present application, with the detection distance dependent upon surrounding materials as well as reader and tag design features. When a tag is within the magnetic field generated by the reader's coil such that the power delivered to the tag is sufficiently high, the tag communicates back to the reader via radio frequency backscattering. The communication is controlled by an integrated circuit on the tag that modulates the tag impedance between two states: matched and unmatched [4]. By recording the reflected radio frequency energy, the reader can "feel" the state of the tag impedance, thus allowing transmission of information from the tag to the reader. The tag's integrated circuit thus generates a byte stream to the reader. For each tag, the integrated circuit can be programmed to generate a unique pattern of high and low impedances (a unique byte stream), allowing tags on different socks to be distinguished from each other [4]. The reader then demodulates the backscattered signals and transmits the data to a data storage medium.

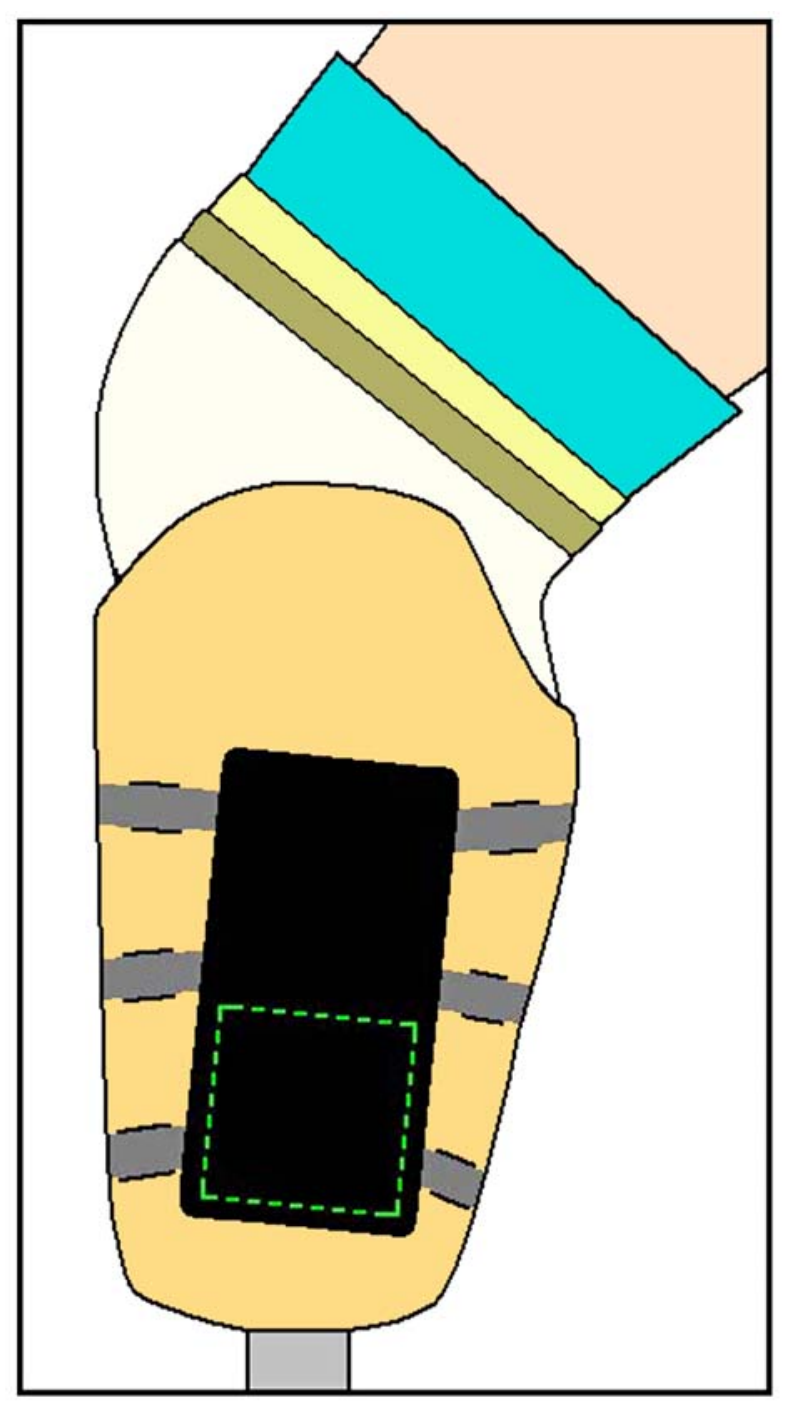

Figure 1.

Sock monitor on socket. Prototype was mounted on anterior lateral surface with Velcro straps. Reader antenna (dashed line) was contained within box in this prototype.

We used a modular HF RFID reader (M4, SkyeTek; Denver, Colorado) with a microcontroller (MSP430F1611, Texas Instruments; Dallas, Texas), oscillator, secure digital (SD) card (31198-2GBCSTA, Kodak; Rochester, New York), card connector (Olimex; Plovdiv, Bulgaria), $9 \mathrm{~V}$ battery, and antenna to create a device that continuously monitored sock presence and stored data to the card (Figure 2). Upon depressing a power switch, the microcontroller repeatedly executed a simple collect-and-store sequence. Seven times each $2 \mathrm{~s}$, the microcontroller sent a command to the RFID module requesting an inventory of tags in the 


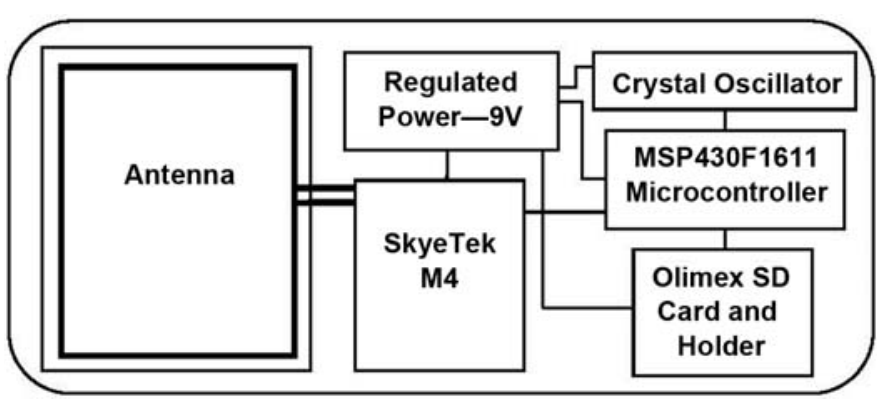

Figure 2.

System layout. Components were housed within box mounted to external socket surface. SD = secure digital.

range of the antenna. A tag, with its own unique identification (ID) number (byte stream) and of surface area approximately that of a dime, was attached to each sock tested. The tags were flexible, similar to those currently used in the garment industry for inventory tracking. The RFID module responded with the tag ID numbers from the tag inventory (any tag recognized during the seven sequences was recorded once), and the list was stored to the microcontroller. Before the microcontroller sent another inventory command, it saved the tag inventory byte stream to a text file on the SD card. With this strategy, data on the SD card was constantly updated, thus ensuring no data were lost if the system was turned off. Using a 9 V/1,200 mAh battery, this system was capable of operating continuously for approximately $10 \mathrm{~h}$.

\section{Bench Testing}

We performed bench tests to evaluate the distance over which the reader detected tags as well as sensitivity to tag/reader orientation and materials near the unit.

\section{Unobstructed Detection Distance}

We tested five different tag sizes to determine the distance range over which tags could be detected. Four of the tags were rectangular and one was circular (Table). Tags were acquired from Avery Dennison (Pasadena, California) and Texas Instruments (Dallas, Texas). All tags used coil antenna geometries.

We placed the sock monitor on a laboratory table and positioned a wooden ruler vertically adjacent to the reader antenna. We held a test tag parallel and centered to the face of the reader antenna several millimeters above its surface until communication was established between the tag and the reader. Then, we slowly moved the tag away from the reader $(15 \mathrm{~mm} / 10 \mathrm{~s})$ until the connection
Table.

Radio frequency identification tags used for unobstructed detection distance tests.

\begin{tabular}{cclc}
\hline Tag ID & $\begin{array}{c}\text { Manufacturer- } \\
\text { Model }\end{array}$ & Shape & $\begin{array}{c}\text { Antenna } \\
\text { Dimensions } \\
\text { Length } \times \text { Width } \\
(\mathbf{c m})\end{array}$ \\
\hline 1 & AD-714 & Rectangle & $7.1 \times 4.0$ \\
2 & AD-709 & Rectangle & $4.0 \times 4.0$ \\
3 & TI-RI-I11- & Rectangle & $3.5 \times 3.5$ \\
4 & AD-720 & Circle & 3.3 (diameter) \\
5 & AD-730 & Rectangle & $2.8 \times 1.0$ \\
\hline $\begin{array}{l}\text { AD }=\text { Avery Dennison (Pasadena, California), TI }=\text { Texas Instruments (Dallas, } \\
\text { Texas). }\end{array}$ & & \\
\hline \hline
\end{tabular}

was lost. We made several slow up/down oscillations of approximately $1 \mathrm{~mm}$ amplitude such that the tag was in communication at the trough and out of communication at the peak. We noted the trough as the maximum read distance for that tag. We tested three tags of each type and recorded the mean maximum read distance.

Results showed that read distance increased with surface area (Figure 3) and ranged from $5.6 \mathrm{~cm}$ for the smallest tag (\#5) to $12.7 \mathrm{~cm}$ for the largest tag (\#1). Tag detection was independent of sock material or sock thickness for thickness up to at least $1 \mathrm{~cm}$.

\section{Detection in Presence of Socket Materials and Human Tissue}

We tested the effect on performance of different materials in the reader/tag environment by creating a test model. The materials tested were intended to reflect those the sock monitor would be exposed to in clinical use. We filled a plastic bag with a mixture of salt and water so that it had an equivalent conductivity to human muscle tissue [5]. The bag of saltwater was inserted into a prosthetic elastomeric liner, the liner was covered with a 3-ply cotton sock, and then the assembly was inserted into a prosthetic socket. Two sockets were tested: a PETG (glycol-modified polyethylene terephthalate) socket and a composite socket that included three layers of carbon fiber. We tested these sockets to determine whether performance was dependent on socket material. These socket materials were selected because they presented low and high obstructive properties for RFID transmission. A different reader antenna, made of copper traces printed on a flexible substrate (antenna surface area $24.0 \mathrm{~cm}^{2}$ ), was used instead of the stiff-backed antenna included with the SkyeTek kit 
JRRD, Volume 49, Number 8, 2012

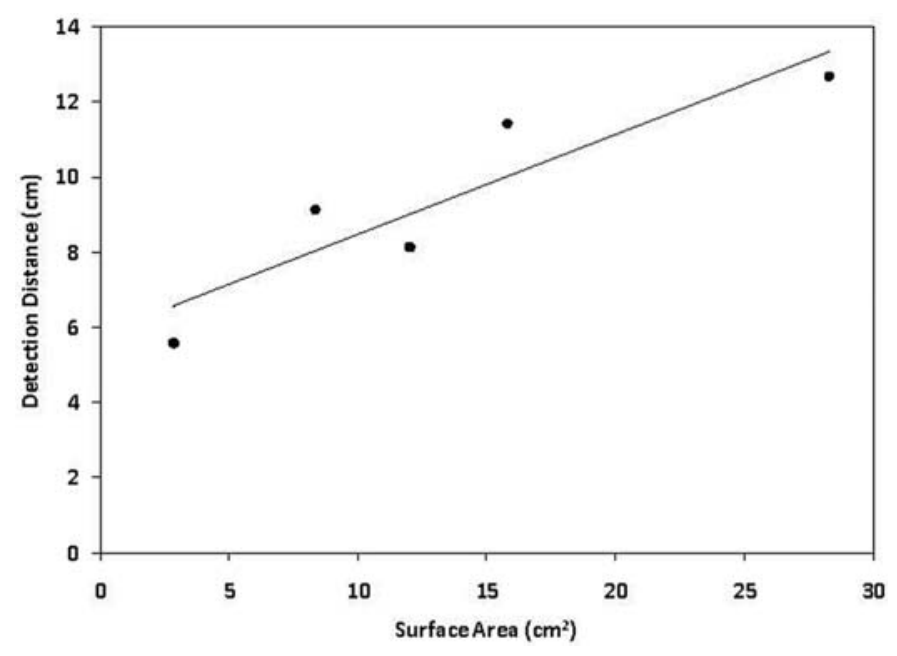

Figure 3.

Unobstructed read distance results. Maximum read distance for five tags tested was approximately proportional to surface area of tag's antenna.

because the antenna needed to be flexible to fit on the inside surface of a prosthetic socket.

Four small tags (\#5 in Table) in a $2 \times 2$ matrix were used as a test article. We chose small tags because they fit within the area of the reader's antenna without overlap, and we used four tags because that was the maximum number possible within this space. The test article was inserted into the test socket between the sock and liner. In a static reliability test, we positioned the antenna outside of the socket (at about three-fourths of its detection range) with the test article centered and monitored for a $30 \mathrm{~s}$ interval. In a dynamic detection test, we slowly moved the antenna in an area sweep about the face of the tag to determine whether the maximum read count (number of socks detected) was the same as in the static reliability tests.

We found for all tests that if all four tags were detected in a given test setup, the first detection of all tags occurred within the first $9 \mathrm{~s}$ of sampling. During static reliability testing, all four tags were detected using the polymer socket but only two were detected using the carbon fiber socket, likely because of detuning of the RFID antennae from the presence of the carbon fiber.

We created additional test configurations to assess material presence behind and in front of as opposed to between the reader antenna and RFID tag. First, we placed the antenna on a loose sheet of carbon fiber weave
(0.3 mm thickness), and then we placed the test article on the antenna. In the second configuration, we placed the test article between a sock and liner that had been cut at the distal end so as to fit over the proximal aspect of the lower leg (over the gastrocnemius area) of a nondisabled person. The flexible reader antenna was placed over the outside of the liner. We tested with and without the carbon fiber weave over the antenna. A third configuration was identical to the second except that bony human tissue (the anterior medial tibial area of the proximal lower leg of a nondisabled person) was used.

Results were very sensitive to slight position changes of the test materials relative to the antenna, a finding expected to reflect inhomogeneities in the test materials. Quantitative test results demonstrated too much scatter in the data to be statistically meaningful. Qualitatively, we observed that the three materials had a moderate to strong influence toward decreasing the number of tags detected. In order of greatest to least influence these three materials were carbon fiber, fleshy human tissue (gastrocnemius), and bony human tissue (tibia).

\section{Preliminary Studies on Participants with Transtibial Amputation}

\section{Subjects}

We collected data on three participants with unilateral transtibial amputation. Subjects were included in the study if they had a transtibial limb amputation more than 18 mo prior, they were K-2 (Medicare Functional Classification Level [6]) or higher level ambulators, and their socket fit as deemed by the research prosthetist was acceptable for regular use. Subjects were excluded if they had current skin breakdown.

Subject \#1 was a 52 yr old male who had a right transtibial amputation 2 yr prior because of osteomyelitis. He had pitting edema in his contralateral limb at level 3+ on a scale of 0 to $3+$. He used a patellar-tendon bearing (PTB) endoskeletal prosthesis; a composite socket with one layer of Dacron, two layers of Kevlar, and four layers of nylon; a $3 \mathrm{~mm}$ Ossur Iceross Comfort liner; and a Seattle LightFoot. He claimed to use one 3-ply and one 5 -ply wool sock every day, all day with no changes. The research practitioner suspected that he did not change socks because of his poor sensation. His prosthesis was suspended by a distal lock and pin attachment, but the socket may have been loose fitting around the proximal section because socks were not added to take up volume 
loss over the day. Because of the long, large shape of his residual limb, the high surface area may have helped accommodate for the lack of sock-ply.

Subject \#2 was a 63 yr old male with transtibial amputation from a traumatic accident 8 yr prior. He had a conical residual limb. He was very aware of his prosthetic fit and knew when he should add a sock for better fit. He used a PTB endoskeletal prosthesis, a polypropylene socket, a $6 \mathrm{~mm}$ Alpha liner, lock and pin suspension, and a Luxon Max dynamic response prosthetic foot. His residual limb volume fluctuated during the day very little, and he added a 1-ply cotton sock only during extreme activity.

Subject \#3 was a 48 yr old female with right transtibial amputation due to multiple burn injuries from a house fire more than $10 \mathrm{yr}$ prior. She had a very short conical residual limb with scar tissue from traumatic burns. There were signs of too much distal end contact with firm callous and pressure marks on the distal end of the tibia, distal patella, and medial condyle. She was delivered a PTB-endoskeletal prosthesis 15 mo prior, with a $3 \mathrm{~mm}$ Ossur Iceross Comfort liner with lock and pin suspension and Seattle Carbon Lightfoot. Her composite socket was made of one layer of Dacron, six layers of Nyglass stockinette, two layers of carbon fiber weave, and two layers of nylon stockinette. In this prosthesis, her volume fluctuated throughout the day, but never more than a 1-ply sock at a time.

\section{Protocol}

The tags were placed on the insides of the socks using paper tape, and subjects donned the socks such that the RFID tags were over the anterior lateral distal region of the residual limb. We found that this location produced the most reliable measurements, in part because this area was relatively flat and the box housing the reader and antenna was mechanically stable at this location and did not interfere with gait. Subjects used blend or wool socks of 1-, 2-, or 3-ply. When socks were added, as indicated by the protocol described in this section, the research practitioner selected sock ply based on what was comfortable for the subject.

We conducted two different test protocols: (1) Fitting test: Data were collected during donning, sitting, standing, and doffing. We performed tests sequentially with the subject donning an additional RFID-tagged sock on the residual limb for each successive test. Tests were conducted until the subject deemed the number of socks to cause an uncomfortable fit or the research prosthetist felt that additional socks put the subject's limb at risk of injury. (2) Activity test: Wearing the number of socks deemed comfortable and appropriate, data were collected continuously during donning, standing, walking, stairclimbing, lateral shifting, and doffing. Subject \#2 conducted additional tests on different walking surfacesgrass, gravel, and an inclined surface.

While data collection on Subjects \#1 and \#2 was successful, Subject \#3's socket inhibited communication between the reader and tags. This subject's socket, unlike those of Subjects \#1 and \#2, had several carbon fiber layers embedded within its lamination. We tested additional sockets available in the laboratory from prior research projects and found that some sockets limited reader/tag communication and others did not. In general, the greater the number of carbon fiber layers, the greater the interference to reader/tag communication.

\section{Results}

For Subjects \#1 and \#2, for both the fitting tests and the activity tests, we found it necessary at the outset of the test session to carefully adjust the position and orientation of the box holding the reader and antenna so as to achieve effective reader/tag communication. There was a narrow range of approximately $\pm 0.5 \mathrm{~cm}$ from the center of the tags within which reader/tag communication was achieved. Once proper position and orientation were established, however, no adjustment was needed for the rest of the test session if three or fewer tags were worn.

Results from the fitting tests for Subjects \#1 and \#2 demonstrated that the system recognized socks well when one or two socks were worn, moderately well when three socks were worn, and poorly when four socks were worn (Figure 4(a) and (b)). We found that with three or more socks, the system identified only some of the socks at times and then at other times identified all of the socks. When Subject \#1 wore four socks, despite numerous attempts at adjusting the socks to avoid overlapping RFID tags with each other, we were not able to reliably detect more than three socks (Figure 4(b)). These results suggest that when tags were adjacent to one another, a “collision” problem occurred. In RFID terminology, collision means that tags interfere such that some tags mask detection of other tags.

Over the course of a 7 min activity session for Subject \#1, the system performed well in that it provided a continuous uninterrupted output signal. It correctly recognized that the subject wore three socks, albeit with a 
JRRD, Volume 49, Number 8, 2012
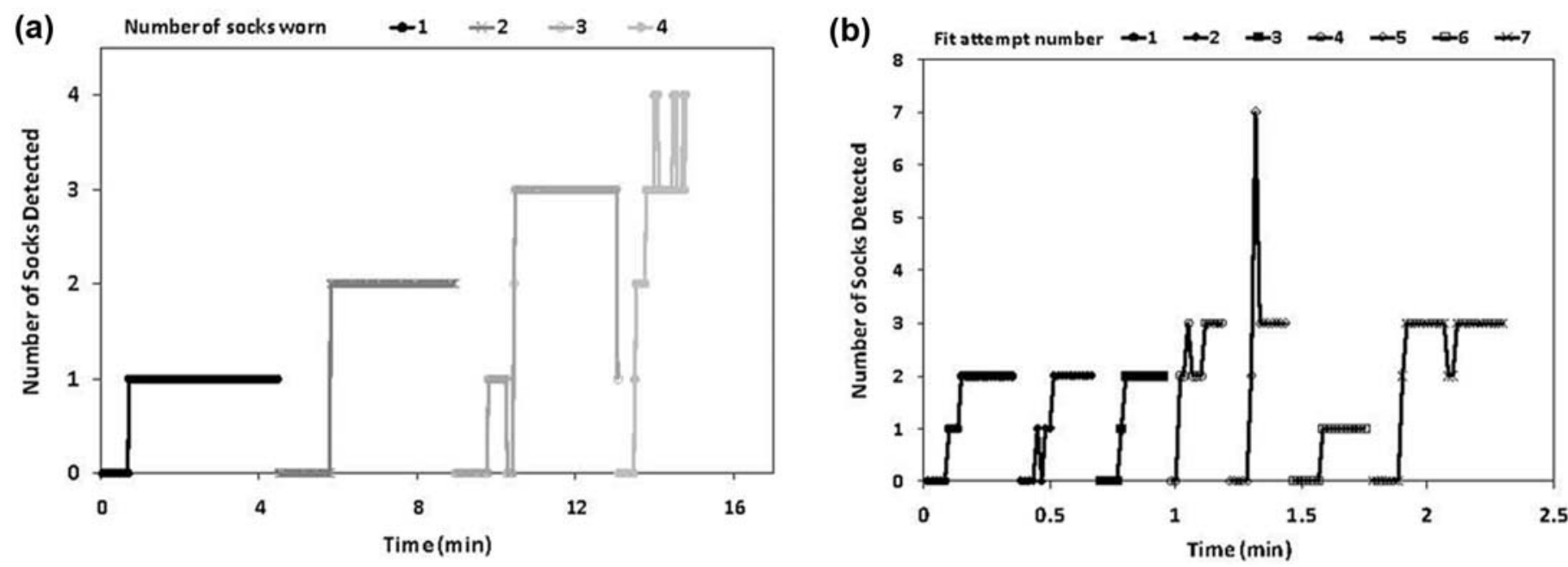

Figure 4.

Fitting test results. (a) Subject \#2: During each of four testing intervals, subject wore number of socks indicated (1, 2, 3, or 4). (b) Subject \#1 wearing four socks. Seven attempts were made, making adjustments to antenna or tag positions, to detect all four socks but none was successful. System was turned off between tests while subject doffed socket; thus, time axes represent cumulative time during which data were collected.

few errors (Figure 5(a)). Intermittent errors occurred when limb-socket displacement (pistoning) was likely elevated-right after donning, walking down stairs, and as the prosthesis was prepared for doffing. The intermittent errors were expected to be the result of several factors-the tag moved too far away from the reader antenna to be powered, there were collision problems, processing filters embedded within the M4 reader module were inappropriate for our application, and excessive angulation of the plane of the tag antenna relative to that of the reader antenna was present. When Subject \#1 wore four socks, detection deteriorated (Figure 5(b)). Typically, the reader detected only three of the four socks.

The sock monitor performed better during activity tests on Subject \#2 than on Subject \#1. During donning, standing, walking, stair-climbing, lateral shifting, and doffing over a $1 \mathrm{hr}$ period, the system consistently detected all three socks (Figure 6). In addition, walking on other surfaces, including grass, gravel, and an inclined surface, did not distort performance.

\section{DISCUSSION}

A sock monitor may prove useful for prosthetic fitting by providing quantitative information to the practi- tioner between clinical visits about how many socks a patient wears and when sock changes are made. Potentially, the technology could be used with a feedback system (alarm, message to smartphone) to alert patients when they need to add a sock. The efforts described here demonstrate that a sock monitor based on RFID technology is possible, but there are challenges that need to be overcome for it to operate as a reliable clinical device. We present here explanations of the challenges and potential solutions.

\section{Socket Material}

Carbon fiber is a semiconductive material, having a conductivity of about 1/900th that of copper, though the exact value depends on the number of carbon fibers per braid and other factors [5]. Carbon fiber-mesh layers within the socket prevented reader/tag communication because the carbon fiber altered the resonant frequency of the reader antenna away from $13.56 \mathrm{MHz}$ and/or interrupted its magnetic field so that the tags were not powered. Such interference from surrounding materials is a recognized problem in RFID technology [3]. To solve this problem, the engineer typically modifies the reader antenna so that it tunes to $13.56 \mathrm{MHz}$ with the interfering material present and/or enhances the magnetic field strength, for example by adding more windings to the reader's coil. An 
(a)

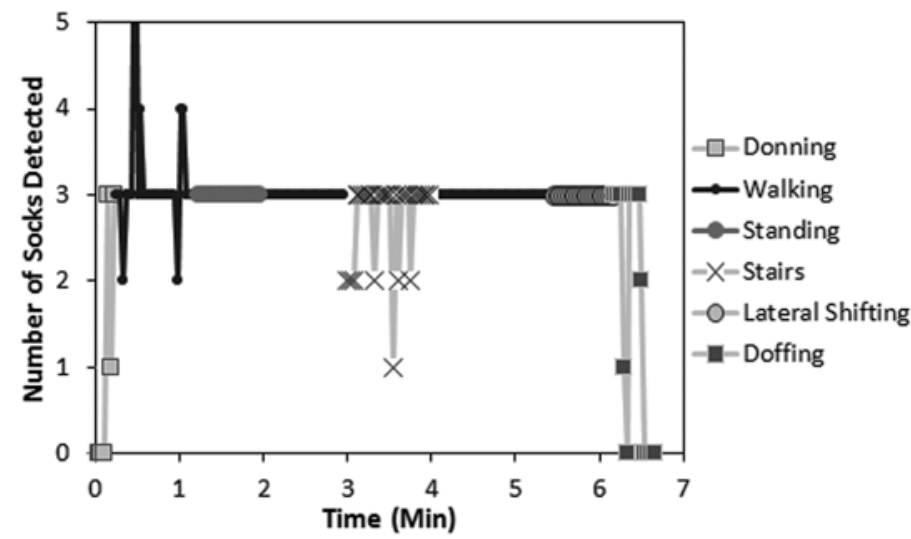

(b)

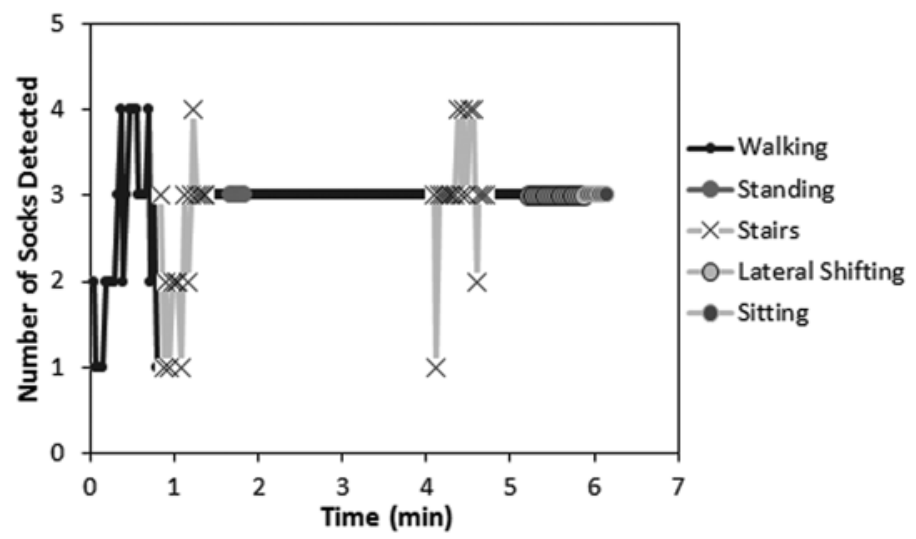

Figure 5.

Activity test results: Subject \#1. Subject wore (a) three socks and (b) four socks. Reader detected all socks well when three socks were worn (a), but failed to detect all socks when four socks were worn (b).

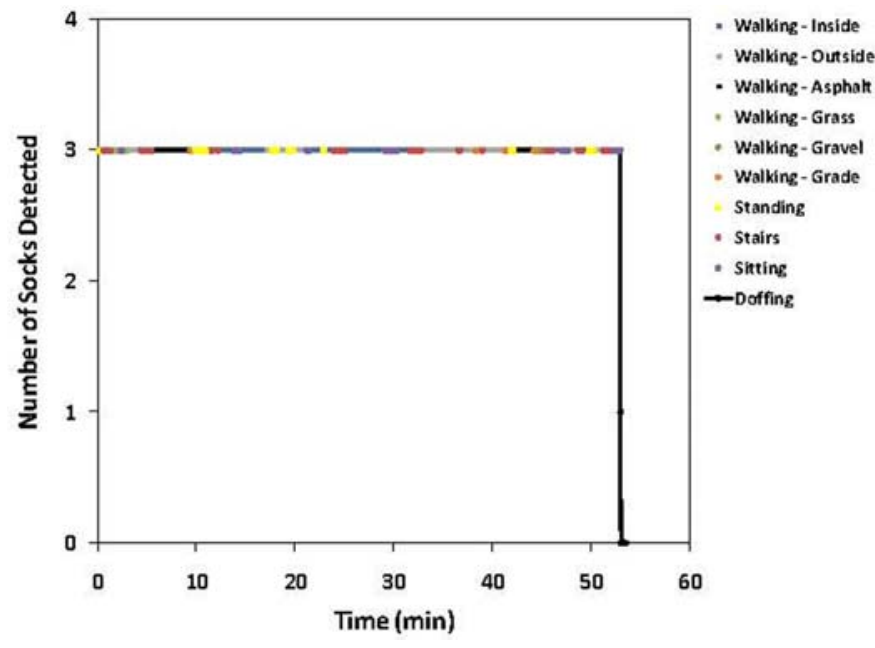

Figure 6.

Activity test results: Subject \#2. Subject wore three socks during entire test while he walked on different surfaces, stood, climbed stairs, or sat. Reader detected sock presence well for all activities.

alternative strategy that avoids the need to transmit through the carbon fiber material altogether is to place the reader antenna on the inside socket surface, within a flex circuit, for example, or within the wall of the socket. However, even though the carbon fiber is not between the reader and tag, it can still cause detection problems, as demonstrated in our bench tests. The reader antenna must be carefully designed to prevent interference.

\section{Angle of Tag Relative to Reader Antenna}

With HF RFID, the reader powers the tag via the magnetic field generated by its antenna coil. It will power the tag only when the plane of the tag's antenna coil is parallel with that of the reader's. Ultra-high-frequency (UHF) RFID overcomes this problem by generating electromagnetic fields instead of magnetic fields. In electromagnetic fields, electrical and magnetic fields orthogonal to each other are generated via a feed point on the antenna, generating fields in multiple directions [4]. The single direction limitation of HF RFID is avoided. Because of this capability, with UHF the tags do not have the restriction of being positioned in parallel planes with the reader antenna, and thus may be more effective at overcoming tag antenna to reader antenna orientation problems for the present application.

\section{Collision}

RFID readers may have difficulty detecting overlapping tags because one tag may shield power to other tags or distort radio frequency backscatter from other tags to the reader. In recent years, anticollision algorithms have been generated for UHF RFID systems that help overcome this problem [7]. The Gen2 tag used with UHF is, in part, a result of these efforts. Tag size, shape, and position could also be adjusted to overcome collision problems.

\section{Large Displacements (Pistoning)}

Subject \#1's excessive pistoning compared with Subject \#2's was likely the primary reason why sock detection 
was less effective for Subject \#1 than Subject \#2. For HF RFID, when the tag moves outside of the closed contour formed by the coil generating the magnetic field, the tag will not be powered and thus will not be detected. This problem can be overcome by enlarging the size of the antenna or using more antennae. However, these modifications will come at the expense of increased power consumption. UHF overcomes this problem because UHF magnetic fields, as described in the previous section, are not limited to a single direction. Also, the reader powers the tags via both electric field coupling (at greater distances) as well as magnetic field coupling. The distance range over which UHF detects tags is greater than HF, making it possible that tags on socks outside of the socket, for example on a table near the prosthesis, are detected. Thus if UHF is implemented, efforts may need to be taken to eliminate detection of such tags or recognize that the prosthesis is not being worn so as to avoid confusion in clinical interpretation of the data.

\section{Power}

To extend from the design described here that operates for $10 \mathrm{~h}$ of continuous use to a longer-term monitoring tool (e.g., months), a power management strategy will need to be implemented. Adding a thin in-socket pressure sensor (e.g., force sensing resistor (FSR), capacitive sensor, or optical pressure sensor) to the inside surface of the posterior socket wall, for example, to identify when the socket is doffed and feeding the signal into the microcontroller would allow power to be turned off when the prosthesis was not worn. Pressure sensors can now be embedded within flex circuits; thus, they can be positioned nonobtrusively inside the socket against the posterior socket wall. This technology has been used for socket pressure monitoring in research investigations [8]. Since only sock changes need to be monitored to characterize a patient's sock use, not continuous sock presence as performed in this study, the system could be turned off except when in-socket pressures indicated a change from a doffed to donned state occurs. In addition, FSR data coupled with sock monitor information may provide the practitioner with insight into how sock use relates to patient activities (e.g., standing, walking, sitting), environment (e.g., uphill climbing), and health (e.g., balance), more information than just sock presence alone. Such information could provide additional insight useful to prescription.
Once the challenges described here are overcome, a key step is to collect data on people wearing prosthetic limbs to determine whether and how sock use data are relevant to patient care. Answering the following questions may provide insight: How often do people add a sock? How regular are their sock-addition practices? How do results relate to patient comfort and satisfaction with the prosthesis? How much do decisions to change sock ply relate to a person's subsequent activity? As active RFID tags become available, tags that have sensing elements within them (e.g., temperature, sweating, displacement, or force sensors) may allow additional information to be collected and provided to the practitioner and patient. Potentially, feedback systems can be developed to alert patients when they need to add a sock. Other applications, for example monitoring sock use in diabetic foot patients, may also provide clinically useful insight to enhance patient care and quality of life.

\section{CONCLUSIONS}

RFID technology can be effectively used to monitor sock presence in people using prosthetic limbs. While HF RFID detected sock presence, UHF RFID may be more effective for this application so as to overcome problems with collision, tag orientation, pistoning, and interference. Once a small portable device is created, clinical investigations need to be conducted to determine whether and how sock use data help the practitioner diagnose the patient's status and needs and create an effective prosthesis prescription. Potentially, the sock monitor can be extended into a clinical feedback device to tell a patient via alarm, smartphone message, or some other means when a sock needs to be added or removed.

\section{ACKNOWLEDGMENTS}

\section{Author Contributions:}

Study concept and design: J. E. Sanders, B. P. Otis. Acquisition of data: R. Murthy, J. C. Cagle, R. H. Phillips. Analysis and interpretation of data: J. E. Sanders, J. C. Cagle, K. J. Allyn, R. H. Phillips.

Drafting of manuscript: J. E. Sanders, J. C. Cagle.

Critical revision of manuscript for important intellectual content:

R. H. Phillips, R. Murthy, K. J. Allyn, B. P. Otis.

Obtained funding: J. E. Sanders.

Study supervision: J. E. Sanders, J. C. Cagle, B. P. Otis. 
Financial Disclosures: The authors have declared that no competing interests exist.

Funding/Support: This material was based on work supported by the National Institutes of Health (grant HD069387-01).

Additional Contributions: We appreciate input from Pavel Nitkin, $\mathrm{PhD}$, from Intermec (Everett, Washington) concerning RFID technology.

Institutional Review: All procedures were approved by a University of Washington Institutional Review Board, and informed consent was obtained before study procedures were initiated.

Participant Follow-Up: The authors do not plan to notify the study subjects of the publication of this article.

\section{REFERENCES}

1. Highsmith JM, Kahle JT. Prosthetic socks: simple, lowcost, helpful ways to protect your skin [Internet]. In Motion. 2006;16(2). Available from: http://www.amputeecoalition.org/easyread/inmotion/mar apr 06/ prosthetic socks-ez.html.

2. Sanders JE, Fatone S. Residual limb volume change: systematic review of measurement and management. J Rehabil Res Dev. 2011;48(8):949-86. [PMID:22068373] http://dx.doi.org/10.1682/JRRD.2010.09.0189

3. Uddin J, Reaz MB, Hasan MA, Nordin AN, Ibrahimy MI, Ali MA. UHF RFID antenna architectures and applications. Sci Res Essays. 2010;5(10):1033-51.

4. Nikitin PV, Rao KV, Lazar S. An overview of near field UHF RFID. In: Proceedings of IEEE International Conference on RFID. 2007; Grapevine, Texas. Piscataway (NJ): IEEE: 2007. p. 167-74.

5. MatWeb Material Property Data [Internet]. MatLab, LLC [cited 2012 Sep 12]. Available from: http://www.MatWeb.com.
6. Medicare region $\mathrm{C}$ durable medical equipment prosthetics orthotic supplier (DMEPOS) manual. Columbia (SC): Palmetto GBA; 2005; 53.5-53.6.

7. Klair DK, Chin K-W, Raad R. A survey and tutorial of RFID anti-collision protocols. IEEE Communications Surveys \& Tutorials. 2010;12(3):400-21. http://dx.doi.org/10.1109/SURV.2010.031810.00037

8. Dumbleton T, Buis AW, McFadyen A, McHugh BF, McKay G, Murray KD, Sexton S. Dynamic interface pressure distributions of two transtibial prosthetic socket concepts. J Rehabil Res Dev. 2009;46(3):405-15.

[PMID:19675992]

http://dx.doi.org/10.1682/JRRD.2008.01.0015

Submitted for publication September 14, 2011. Accepted in revised form January 5, 2012.

This article and any supplemental material should be cited as follows:

Sanders JE, Murthy R, Cagle JC, Allyn KJ, Phillips RH, Otis BP. Device to monitor sock use in people using prosthetic limbs: Technical report. J Rehabil Res Dev. 2012; 49(8):1229-38.

http://dx.doi.org/10.1682/JRRD.2011.09.0169

Research ID: Joan E. Sanders, PhD: E-8204-2011

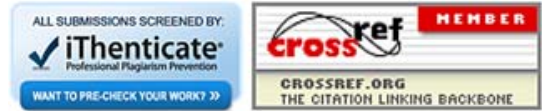


